\title{
Avaliação eletromiográfica dos músculos do membro superior de indivíduos hemiparéticos com uso da Terapia do Espelho
}

\section{Electromyography of the upper limb muscles of patients with hemiparesis using Mirror Therapy}

\author{
Ana Cláudia Tavares Rodrigues ${ }^{1}$, Flávia Roberta Faganello Navega ${ }^{2}$ \\ http://dx.doi.org/10.11606/issn.2238-6149.v27i3p278-288
}

\begin{abstract}
Rodrigues ACT, Faganello-Navega FR. Avaliação eletromiográfica dos músculos do membro superior de indivíduos hemiparéticos com uso da Terapia do Espelho. Rev Ter Ocup Univ São Paulo. 2016 set.-dez.;27(3):278-88.
\end{abstract}

RESUMO: O objetivo deste estudo foi analisar a atividade elétrica muscular durante a realização da Terapia do Espelho no membro superior parético de indivíduos com sequela de AVE. Trata-se de um estudo de caráter quantitativo. Participaram desta pesquisa dez usuários do Centro de Reabilitação SORRI-BAURU, com histórico de AVE há pelo menos seis meses, com sequela de hemiparesia. A coleta de dados foi constituída pela aplicação da Escala Modificada de Ashworth e avaliação eletromiográfica dos seguintes músculos do membro superior acometido: abdutor curto do polegar, flexor radial do carpo, extensor ulnar do carpo, bíceps e tríceps. A análise do sinal EMG foi realizada em rotinas específicas desenvolvidas em ambiente Matlab (Mathworks ${ }^{\circledR}$ ) e a análise estatística feita através do teste ANOVA para medidas repetidas, sendo adotado o nível de significância de $\mathrm{p}<0,05$. Foram encontradas diferenças significativas nos dados referentes aos músculos abdutor curto do polegar $(\mathrm{p}=0,005)$; flexor radial do carpo $(\mathrm{p}=0,032)$; bíceps $(\mathrm{p}=0,002)$ e tríceps braquial $(\mathrm{p}=0,024)$, provavelmente, devido a uma adequação do tônus durante a realização da Terapia do Espelho, porém, ainda é necessária a realização de estudos que comparem estes dados pré e pósintervenção com o uso da técnica, em busca de uma recuperação motora mais efetiva do membro acometido.

DESCRITORES: Acidente vascular encefálico; Eletromiografia; Terapia ocupacional.
Rodrigues ACT, Faganello-Navega FR. Electromyography of the upper limb muscles of patients with hemiparesis using Mirror Therapy. Rev Ter Ocup Univ São Paulo. 2016 Sept.Dec.;27(3):278-88.

ABSTRACT: The aim of this study was to analyze muscle electrical activity during the performance of the Mirror Therapy with the upper limb of paretic subjects with stroke. This is a quantitative character study. Participated in this survey ten users of SORRI - BAURU Rehabilitation Center with stroke history for at least six months with hemiparesis sequel. Data collection was formed by the application of the Modified Ashworth Scale and electromyographic evaluation of the affected upper limb in the following muscles: abductor pollicis brevis, flexor carpi radialis, extensor carpi ulnaris, biceps and triceps. The analysis of the EMG signal was performed in specific routines developed in Matlab (Mathworks ${ }^{\circledR}$ ) and statistical analysis was applied using ANOVA for repeated measures, adopting the significance level of $p<0.05$. Significant differences were found on the data for abductor pollicis brevis $(p=0.005)$; flexor carpi radialis $(p=0.032)$; biceps $(p=0.002)$ and triceps $(\mathrm{p}=0.024)$, probably due to an adjustment of tone during the course of mirror therapy, however, it is still necessary to carry out studies comparing these data before and after the intervention using the technique in search of a more effective motor recovery member affected.

KEYWORDS: Stroke; electromyography; Occupational therapy.

\footnotetext{
Artigo originado de dissertação de Mestrado apresentada ao Instituto de Biociências do Campus de Rio Claro, Universidade Estadual Paulista (UNESP), como parte dos requisitos para a obtenção do título de Mestre, Programa de Pós Graduação em Desenvolvimento Humano e Tecnologias.

1. Mestre em Desenvolvimento Humano e Tecnologias - Universidade Estadual Paulista (UNESP), campus de Rio Claro.

2. Docente do curso de Fisioterapia da Universidade Estadual Paulista (UNESP), campus de Marília e do programa de pós-graduação

Stricto Sensu em Desenvolvimento Humano e Tecnologias - Universidade Estadual Paulista (UNESP), campus de Rio Claro.

Endereço para correspondência: DEFITO - Departamento de Fisioterapia e Terapia Ocupacional. Universidade Estadual Paulista (UNESP) - campus Marília. Av. Hygino Muzzi Filho, 737, Bairro Mirante, Marília, SP. CEP: 17525-000. E-mails: cacaautavares@gmail. com / frfaganello@marilia.unesp.br
} 


\section{INTRODUÇÃO}

o longo dos anos, podemos observar um
aumento considerável na incidência de
Acidente Vascular Encefálico (AVE) no mundo todo, com crescimento do número de indivíduos com sequelas e disfunções neurológicas ${ }^{1}$.

Dentre os comprometimentos causados pelo AVE, a hemiparesia no lado contralateral da lesão é um dos mais ocorrentes e faz referência à fraqueza muscular ou paralisia motora parcial em um hemicorpo ${ }^{2}$. A hemiparesia pode ocasionar prejuízos na coordenação motora fina e global do membro superior, com déficits na amplitude de movimento, alcance, preensão e função ${ }^{3}$, acarretando grande prejuízo na independência durante a realização das atividades cotidianas. Além disso, pode ocorrer a presença da espasticidade, definida como "o aumento, velocidade dependente, do tônus muscular, com exacerbação dos reflexos profundos, decorrente de hiperexcitabilidade do reflexo do estiramento ${ }^{4}$ ".

Uma das formas de se avaliar os déficits causados pela hemiparesia e pela espasticidade no membro superior é por meio da Eletromiografia de Superfície (EMG), que se destaca como um método quantitativo superficial para a avaliação da atividade elétrica muscular ${ }^{5}$.

Estudos de Pereira e Araújo ${ }^{6}$, Campos et al. (2012) e Song e Tong ${ }^{8}$ mostraram que é possível detalhar o comportamento do membro superior parético por meio da EMG de superfície ${ }^{6-8}$ e demonstraram que o sinal eletromiográfico do músculo hipertônico é significativamente diferente em relação ao lado não comprometido, sendo a atividade EMG do lado espástico maior que o lado não comprometido ao repouso e ao reflexo e menor na contração voluntária máxima ${ }^{6}$. Além disso, ocorre maior acometimento da musculatura flexora distal em relação à proximal ${ }^{7}$ e cocontração excessiva entre agonistas e antagonistas que refletem em uma diminuição no desempenho motor geral no membro superior afetado pelo $\mathrm{AVE}^{8}$.

$\mathrm{Na}$ tentativa de minimizar os déficits sensoriomotores e acelerar o processo de recuperação funcional, terapeutas ocupacionais têm utilizado uma técnica na reabilitação do membro superior em pacientes hemiparéticos: a Terapia do Espelho9 ${ }^{9}$ Introduzida por Ramachandran e Rogers em 1992 no tratamento de pacientes com dor fantasma, esta técnica é fundamentada na neuroplasticidade e na ativação dos neurônios-espelho ${ }^{5}$ e consiste em reeducar o cérebro através de uma série de movimentos com o membro não afetado, sendo que este é visto ao espelho como se fosse o membro parético. Com isso, pretende-se que o cérebro simule os movimentos realizados com o membro acometido devido ao reflexo do membro funcional no espelho, com o intuito de melhorar a resposta motora ${ }^{9}$.

Vários estudos buscaram evidenciar os efeitos da Terapia do Espelho na recuperação de indivíduos com hemiparesia, sendo observada melhora na movimentação e função do membro superior acometido em todos eles $^{10-12}$, porém, não foram encontrados, pelas autoras, estudos que mensurem a atividade elétrica muscular no membro superior parético por meio da EMG com o uso da Terapia do Espelho.

Desta forma, justifica-se nosso estudo, pois muitos indivíduos pós-AVE não mantém o uso funcional do membro parético e a EMG é um instrumento de análise quantitativa, o que minimiza a ocorrência de possíveis erros realizados por avaliações subjetivas como questionários e escalas.

Assim, o objetivo do presente estudo foi analisar a atividade elétrica muscular durante a realização da Terapia do Espelho no membro superior parético de indivíduos com sequela de AVE. A hipótese é que a Terapia do Espelho possa ocasionar uma alteração no padrão da atividade elétrica muscular do membro superior parético em comparação à realização dos exercícios propostos sem o uso do espelho.

\section{MATERIAL E MÉTODOS}

Trata-se de um estudo de caráter quantitativo. $\mathrm{O}$ levantamento bibliográfico para subsidiar o referencial teórico foi realizado nas Bases de Dados Pubmed, Lilacs e Bireme. O projeto foi submetido e aprovado pelo Comitê de Ética em Pesquisa envolvendo seres humanos da UNESP Faculdade de Filosofia e Ciências (FFC) - campus Marília através do protocolo número 1111/2014 e pela Plataforma Brasil através do CAAE número 52659216.1.0000.5406.

Este trabalho foi elaborado no período de março de 2014 a março de 2016, durante o período proposto para elaboração de dissertação de Mestrado pelo programa de Desenvolvimento Humano e Tecnologias do Instituto de Biociências da Universidade Estadual Paulista (UNESP), campus de Rio Claro. A coleta de dados foi realizada no primeiro semestre de 2015.

\section{Participantes}

Participaram desta pesquisa dez usuários do Centro de Reabilitação SORRI-BAURU, de ambos os sexos, 
com idade acima de 30 anos, com histórico de Acidente Vascular Encefálico (AVE), com sequela de hemiparesia e que não apresentassem comprometimentos cognitivos.

Os voluntários foram selecionados de acordo com os seguintes critérios: histórico de até dois episódios de AVE, resultando em sequela de hemiparesia em apenas um membro superior; ter, no mínimo, seis meses de lesão; não ter realizado aplicação de toxina botulínica tipo A (TBA) nos músculos do membro superior acometido; ter amplitude de movimento ativa do membro superior comprometido de, no mínimo, 45 graus de flexão e abdução de ombro, 20 graus de extensão de cotovelo, 10 graus de extensão de punho, 10 graus de extensão/abdução do polegar e 10 graus de extensão em articulações metacarpofalangeanas e interfalangeanas em, pelo menos, dois dedos além do polegar13, mensuradas através de goniometria do membro; apresentar, na aplicação do Mini Exame do Estado Mental (Mini Mental), escore maior ao ponto de corte de acordo com a escolaridade do indivíduo (acima de 23 pontos para indivíduos altamente escolarizados, acima de 17 para indivíduos que possuem ensino médio e acima de 13 para indivíduos analfabetos ${ }^{14}$ ).

\section{Procedimentos}

Os participantes foram selecionados por meio do prontuário eletrônico da SORRI-BAURU. Os participantes que aceitaram participar da pesquisa assinaram o termo de consentimento livre e esclarecido, receberam as orientações necessárias acerca da pesquisa e de como ela seria realizada e, então, deram início aos procedimentos práticos.

A coleta de dados foi realizada individualmente em apenas um dia por participante e foi constituída, primeiramente, pela aplicação do Mini Exame do Estado Mental e pela realização da goniometria do membro superior parético, instrumentos utilizados como critérios de elegibilidade. A goniometria foi realizada com a utilização de dois goniômetros: um goniômetro grande (universal) de $20 \mathrm{~cm}$ para mensuração da amplitude de movimento ativa de ombro, cotovelo e punho e um goniômetro para dedos e pequenas articulações para mensuração da amplitude de movimento ativa dos dedos. Para uma melhor padronização, os participantes estavam sentados e foi avaliado o arco de movimento articular na ordem proximal para distal. Todos os voluntários avaliados foram elegíveis à coleta de dados completa.

Em seguida, foi realizada uma anamnese, aplicada a Escala Modificada de Ashworth e realizada a avaliação eletromiográfica do membro superior afetado.

\section{Escala Modificada de Ashworth}

A Escala de Ashworth é uma ferramenta desenvolvida por Ashworth em 1964 para medir o grau de espasticidade de um membro. Visando melhorias no instrumento de avaliação, Bohannon e Smith ${ }^{15}$ modificaram a escala, acrescentando o grau 1+, denominando-a Escala Modificada de Ashworth. Na aplicação, o examinador deve mover ao longo de sua amplitude articular, de forma passiva, o segmento do paciente correspondente ao músculo ou grupo de músculos a ser avaliado e graduar a resistência encontrada ${ }^{15}$.

Atualmente, é a escala mais utilizada para este fim, embora a literatura apresente limitações, dentre elas, sua subjetividade ${ }^{16}$. Como pontos positivos da escala, podemos citar o fato de não necessitar de nenhum equipamento para avaliação, não possuir gastos materiais, ser simples de ser realizada e rápida ${ }^{15,16}$.

Os participantes da pesquisa foram submetidos à aplicação da Escala Modificada de Ashworth no membro superior hemiparético, nos grupos musculares flexores do punho e do cotovelo, buscando uma relação entre o aumento do tônus e os resultados a serem encontrados na EMG com a realização da Terapia do Espelho. Os grupos musculares foram avaliados com o paciente na postura sentada.

\section{Eletromiografia de Superfície}

Para a avaliação da atividade elétrica muscular por meio da EMG de superfície, o participante permaneceu sentado à frente de uma mesa com os membros superiores apoiados.

O registro da atividade eletromiográfica é captado através de um sinal analógico que deve ser convertido para sinal digital para que possa ser visualizado através de um software específico no computador, registro, este, realizado por meio do equipamento Lynx, marca Lynx ${ }^{\circledR}$ Tecnologia Eletrônica Ltda. de 6 canais com frequência de amostragem selecionada em $2000 \mathrm{~Hz}$. Foram utilizados eletrodos descartáveis Solidor, fabricado por Medico Electrodes International ${ }^{\circledR}$, modelo MSGST-06, passivos de $\mathrm{Ag} / \mathrm{AgCl}$, com espuma e gel sólido. Um eletrodo fixado no maléolo lateral do membro inferior contralateral à hemiparesia foi utilizado como referência para reduzir os ruídos durante a aquisição dos sinais.

Foi utilizado também um netbook modelo HP mini 110-3100, fabricante Hewlett-Packard, processador Intel $\AA$ Atom $^{\mathrm{TM}}$ com software específico para registro e análise dos dados da atividade eletromiográfica. 
Foram avaliados os seguintes músculos do membro superior afetado pela hemiparesia: abdutor curto do polegar, flexor radial do carpo, extensor ulnar do carpo, bíceps e tríceps braquial. Para diminuição da impedância, foi realizada a tricotomia com uma lâmina descartável e a assepsia da pele com um algodão embebido em $1,5 \mathrm{ml}$ de álcool etílico $70 \%$ nas regiões onde, posteriormente, seriam posicionados os eletrodos. A colocação e o posicionamento dos eletrodos seguiram as determinações do protocolo Surface Electromyography for the Non-invasive Assessment of Muscles (Seniam) para os músculos abdutor curto do polegar, bíceps e tríceps, de Mogk e $\operatorname{Keir}^{17}$ para o flexor radial do carpo e do Atlas Mioanatômico Eletrônico para Eletromiografia Clínica da CASA Engineering para o músculo extensor ulnar do carpo.

Primeiramente, foi avaliada a ativação eletromiográfica do lado hemiparético em repouso, no período de um minuto. Após, foram realizados cinco tipos de exercícios ativos, com uma série de dez repetições cada, intervaladas por 30 segundos cada, sendo eles:

- Exercício ativo de abdução e adução do polegar, começando da posição aduzida com o antebraço na posição neutra;

- Exercício ativo de flexão e extensão do polegar, começando de um ponto desenhado no indicador do participante, correspondente à extensão, estando o antebraço em supino;

- Exercício ativo de flexão e extensão do punho com tenodese, iniciando a sequência com o punho fletido e os dedos em extensão, com o antebraço na posição neutra;

- Exercício ativo de flexão e extensão do cotovelo, começando com cotovelo estendido e o antebraço em supino.

- Exercício ativo de pronação/supinação, com o cotovelo fletido à $90^{\circ}$, iniciando com o antebraço pronado.

Os exercícios descritos acima foram realizados em quatro situações aleatórias, selecionadas por sorteio antes da coleta de dados com os participantes: realizando os movimentos propostos somente com o lado preservado; realizando os movimentos com ambos os membros ao mesmo tempo; utilizando o espelho apoiado sagitalmente, realizando os movimentos somente com o lado não afetado e, ainda, utilizando o espelho, realizando os exercícios com ambos os membros superiores. A cada etapa, o participante foi orientado sobre a atividade que deveria realizar.

\section{Terapia do Espelho}

Para a realização da eletromiografia aliada à técnica do espelho, foi colocado um espelho comum de $38 \mathrm{~cm} \mathrm{x}$ $48 \mathrm{~cm}$ acoplado a uma base, para melhor posicionamento e segurança, apoiado sagitalmente ao participante e foram dadas as orientações necessárias para que compreendesse que deveria realizar os movimentos com o membro preservado, porém sempre olhando para o reflexo do membro no espelho. Foi realizada familiarização de 10 minutos com padronização dos comandos verbais e a mesma sequência de exercícios para todos os participantes, minimizando erros na coleta de dados.

Os resultados esperados na sequência de exercícios realizada com o espelho, solicitando que o participante utilizasse somente o membro não comprometido, seguiram os mesmos pressupostos apontados na literatura sobre a Terapia do Espelho, os quais referem que o reflexo do espelho proporciona ao paciente a visão de sua mão não afetada e da mão refletida, como se fosse o membro afetado, fazendo com que o cérebro do participante entenda que os movimentos realizados pelo membro não comprometido, também estão sendo realizados pelo lado afetado, melhorando a resposta motora.

\section{ANÁLISE DOS DADOS}

A visualização e o processamento digital do sinal eletromiográfico foram realizados pelos programas AqDados 7.02 e AqAnalysis, sinais estes avaliados por meio dos valores da integral da envoltória linear, sendo utilizados como referência os dados de cada músculo durante o repouso.

A análise do sinal EMG foi realizada em rotinas específicas desenvolvidas em ambiente Matlab (Mathworks $\left.{ }^{\circledR}\right)$. O processamento dos dados foi realizado utilizando-se um filtro passa-banda de 20-500Hz. Em seguida, o sinal foi retificado, utilizando o método de retificação por onda inteira. Para criação do envelope linear, foi utilizado um filtro passa-baixa de quarta ordem com frequência de corte de $6 \mathrm{~Hz}$ para suavização do sinal. Todos os procedimentos de registro e análise do sinal eletromiográfico seguiram os Standards for Reporting EMG Data.

Posteriormente, os dados foram agrupados em planilhas no Excel para apresentação em média e desvio padrão. Após, foi aplicada a análise estatística, utilizando o software SPSS ${ }^{\circledR}$ e o teste ANOVA para medidas repetidas, sendo adotado o nível de significância de $p<0,05$. 


\section{RESULTADOS}

Participaram do estudo 10 voluntários com média de idade de $53,4( \pm 13,71)$ anos; destes, $60 \%$ foi do sexo masculino e $40 \%$ do sexo feminino. A Tabela 1 mostra os dados dos voluntários.

De modo a comparar a porcentagem de ativação elétrica muscular durante a realização de exercícios específicos em relação ao repouso dos participantes, primeiramente, foi realizada a coleta eletromiográfica durante o repouso. Os resultados em média e desvio padrão dos músculos avaliados, sendo eles, abdutor curto do polegar, flexor radial do carpo, extensor ulnar do carpo, bíceps braquial e tríceps braquial do membro superior hemiparético dos voluntários estão descritos na Figura 1.

Tabela 1 - Caracterização da amostra. M: masculino; F: feminino; m: meses; a: anos; I: isquêmico; H: hemorrágico; DP: desvio padrão.

\begin{tabular}{c|c|c|c|c|c|c|c|c}
\hline VOLUNTÁRIO & IDADE & SEXO & $\begin{array}{c}\text { TEMPO } \\
\text { AVE }\end{array}$ & $\begin{array}{c}\text { I OU } \\
\text { H }\end{array}$ & $\begin{array}{c}\text { MEMBRO } \\
\text { PARÉTICO }\end{array}$ & $\begin{array}{c}\text { ASHWORTH } \\
\text { BÍCEPS }\end{array}$ & $\begin{array}{c}\text { ASHWORTH } \\
\text { FL. PUNHO }\end{array}$ & $\begin{array}{c}\text { MINI } \\
\text { MENTAL }\end{array}$ \\
\hline 1 & 51 & $\mathrm{M}$ & $6 \mathrm{~m}$ & $\mathrm{H}$ & DIREITO & 1 & 1 & 17 \\
\hline 2 & 71 & $\mathrm{M}$ & $6 \mathrm{~m}$ & $\mathrm{I}$ & ESQUERDO & 0 & 0 & 24 \\
\hline 3 & 67 & $\mathrm{~F}$ & $6 \mathrm{~m}$ & $\mathrm{I}$ & ESQUERDO & $1+$ & $1+$ & 26 \\
\hline 4 & 62 & $\mathrm{M}$ & $7 \mathrm{~m}$ & $\mathrm{I}$ & DIREITO & 0 & 0 & 29 \\
\hline 5 & 35 & $\mathrm{~F}$ & $\begin{array}{c}1 \mathrm{a} 7 \mathrm{~m} \mathrm{e} \\
\mathrm{a} \mathrm{m}\end{array}$ & $\mathrm{I} \mathrm{e} \mathrm{H}$ & DIREITO & 0 & 1 & 28 \\
\hline 6 & 41 & $\mathrm{M}$ & $9 \mathrm{~m}$ & $\mathrm{I}$ & DIREITO & 0 & 0 & 25 \\
\hline 7 & 33 & $\mathrm{~F}$ & $9 \mathrm{~m}$ & $\mathrm{I}$ & DIREITO & 0 & 0 & 17 \\
\hline 8 & 67 & $\mathrm{M}$ & $12 \mathrm{~m}$ & $\mathrm{I}$ & DIREITO & 0 & 0 & 30 \\
\hline 9 & 50 & $\mathrm{M}$ & $1 \mathrm{a} 1 \mathrm{~m}$ & $\mathrm{I}$ & ESQUERDO & 1 & 0 & 28 \\
\hline 10 & 57 & $\mathrm{~F}$ & $1 \mathrm{a} 2 \mathrm{~m}$ & $\mathrm{H}$ & DIREITO & $1+$ & 0 & 30 \\
\hline MÉDIA & $\mathbf{5 3 , 4}$ & \multicolumn{7}{|l}{}
\end{tabular}

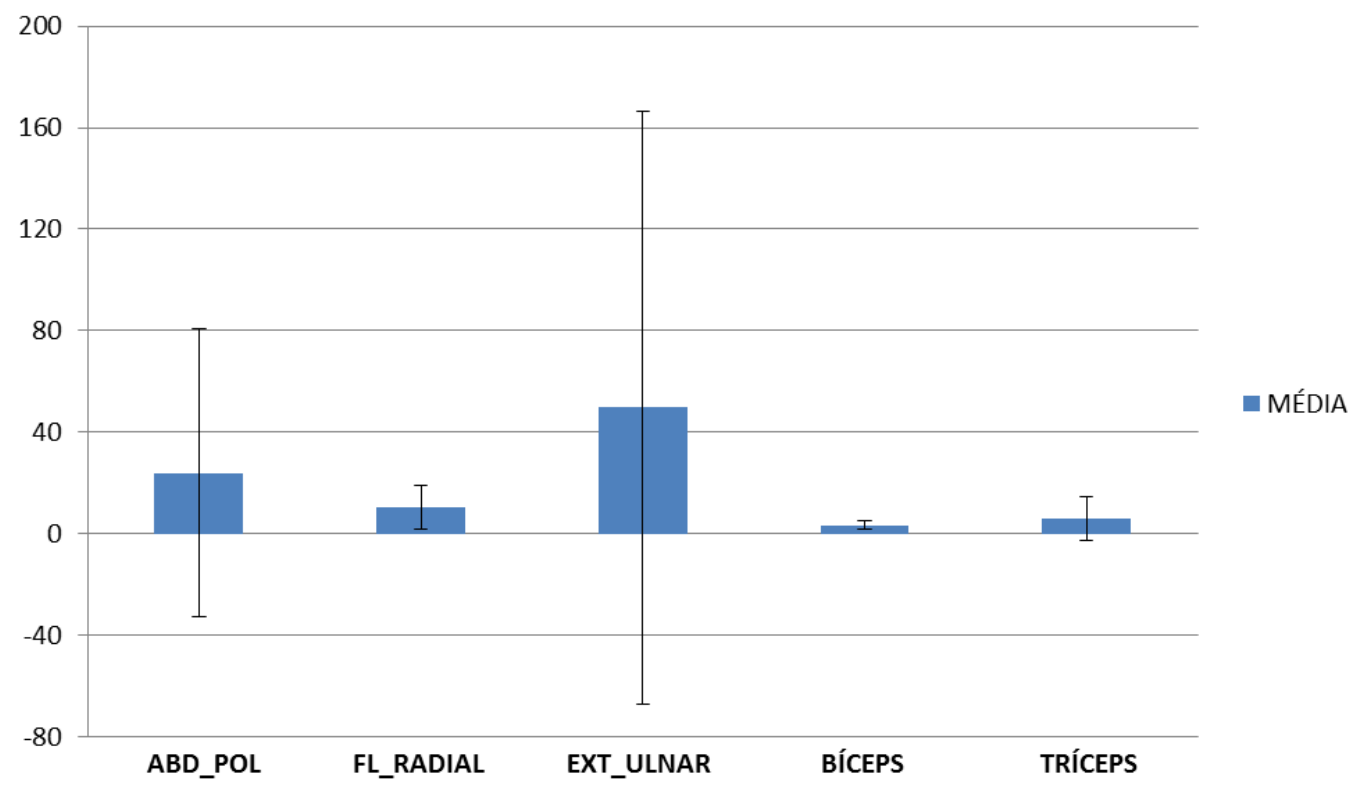

Figura 1 - Média e desvio-padrão da atividade eletromiográfica dos cinco músculos coletados durante o período de repouso dos voluntários. Abd_pol: músculo abdutor do polegar; Fl_radial: músculo flexor radial do carpo; Ext_ulnar: músculo extensor ulnar do carpo 
Para verificar a ativação eletromiográfica dos pacientes, a rotina em ambiente Matlab, além de mostrar os valores absolutos, foi criada de modo a comparar a porcentagem de ativação de cada músculo em cada exercício sobre o valor absoluto do repouso de cada músculo. Sendo assim, a tabela 2 descreve os valores das médias e desvio-padrão de cada músculo avaliado em cada exercício e durante as quatro condições realizadas. $\mathrm{Na}$ média, quando maior que zero, a porcentagem de ativação muscular durante o exercício descrito foi maior do que a porcentagem durante o repouso. Quando menor que zero, a porcentagem de ativação do músculo descrito foi maior durante o período de repouso do que durante a realização do exercício proposto.

Tabela 2 - Valores de média e desvio-padrão da porcentagem de ativação eletromiográfica durante o exercício em relação ao repouso de todos os músculos analisados, em todas as situações. Os valores que apresentaram diferença significativa $(p<0,05)$ estão em negrito na tabela. Abd_pol: músculo abdutor curto do polegar; fl_radial: músculo flexor radial do carpo; ext_ulnar: músculo extensor ulnar do carpo

\begin{tabular}{|c|c|c|c|c|c|c|c|c|c|c|}
\hline & \multicolumn{10}{|c|}{ EXERCÍCIO DE ABDUÇÃO/ADUÇÃO DO POLEGAR } \\
\hline & \multicolumn{5}{|c|}{ MEMBRO NÃO AFETADO - SEM ESPELHO } & \multicolumn{5}{|c|}{ MEMBRO NÃO AFETADO - COM ESPELHO } \\
\hline & abd_pol & fl_radial & ext_ulnar & bíceps & tríceps & abd_pol & fl_radial & ext_ulnar & bíceps & tríceps \\
\hline MÉDIA & $-122,05$ & $-141,04$ & $-248,75$ & $-37,86$ & $-23,73$ & $-150,73$ & $-179,33$ & $-427,84$ & $-27,03$ & $-32,02$ \\
\hline \multirow[t]{3}{*}{ DP } & $\pm 154,17$ & $\pm 274,78$ & $\pm 549,8$ & $\pm 104,77$ & $\pm 58,1$ & $\pm 247,26$ & $\pm 279,84$ & $\pm 738,8$ & $\pm 76,26$ & $\pm 69,09$ \\
\hline & \multicolumn{5}{|c|}{ DUAS MÃOS - SEM ESPELHO } & \multicolumn{5}{|c|}{ DUAS MÃOS - COM ESPELHO } \\
\hline & abd_pol & fl_radial & ext_ulnar & bíceps & tríceps & abd_pol & fl_radial & ext_ulnar & bíceps & tríceps \\
\hline MÉDIA & 48,87 & $-47,6$ & $-2,99$ & 0,45 & $-20,51$ & 34,82 & 0,19 & 31,48 & $-3,81$ & $-13,81$ \\
\hline \multirow[t]{4}{*}{ DP } & $\pm 36,99$ & $\pm 109,31$ & $\pm 121,81$ & $\pm 73,23$ & $\pm 84,2$ & $\pm 101,83$ & $\pm 61,92$ & $\pm 68,72$ & $\pm 76,38$ & $\pm 88,9$ \\
\hline & \multicolumn{10}{|c|}{ EXERCÍCIO DE FLEXÃO/EXTENSÃO DO POLEGAR } \\
\hline & \multicolumn{5}{|c|}{ MEMBRO NÃO AFETADO - SEM ESPELHO } & \multicolumn{5}{|c|}{ MEMBRO NÃO AFETADO - COM ESPELHO } \\
\hline & abd_pol & fl_radial & ext_ulnar & bíceps & tríceps & abd_pol & fl_radial & ext_ulnar & bíceps & tríceps \\
\hline MÉDIA & $-150,7$ & $-279,31$ & $-308,5$ & $-45,72$ & $-59,22$ & $-92,42$ & $-215,93$ & -478 & $-11,91$ & $-17,46$ \\
\hline \multirow[t]{3}{*}{ DP } & $\pm 203,48$ & $\pm 441,66$ & $\pm 810,77$ & $\pm 121,27$ & $\pm 91,08$ & $\pm 182,39$ & $\pm 291,69$ & $\pm 804,02$ & $\pm 54,85$ & $\pm 46,04$ \\
\hline & \multicolumn{4}{|c|}{ DUAS MÃOS - SEM ESPELHO } & \multicolumn{6}{|c|}{ DUAS MÃOS - COM ESPELHO } \\
\hline & abd_pol & fl_radial & ext_ulnar & bíceps & tríceps & abd_pol & fl_radial & ext_ulnar & bíceps & tríceps \\
\hline MÉDIA & 41,26 & $-60,22$ & 22,99 & 45,07 & $-30,7$ & 35,36 & $-109,59$ & $-21,74$ & 26,66 & $-29,85$ \\
\hline \multirow[t]{4}{*}{ DP } & $\pm 57,39$ & $\pm 104,23$ & $\pm 63,83$ & $\pm 39,66$ & $\pm 80,52$ & $\pm 59,91$ & $\pm 192,49$ & $\pm 134,58$ & $\pm 59,52$ & $\pm 127,26$ \\
\hline & \multicolumn{10}{|c|}{ EXERCÍCIO DE FLEXÃO/EXTENSÃO DE PUNHO } \\
\hline & \multicolumn{5}{|c|}{ MEMBRO NÃO AFETADO - SEM ESPELHO } & \multicolumn{5}{|c|}{ MEMBRO NÃO AFETADO - COM ESPELHO } \\
\hline & abd_pol & fl_radial & ext_ulnar & bíceps & tríceps & abd_pol & fl_radial & ext_ulnar & bíceps & tríceps \\
\hline MÉDIA & $-79,07$ & $-135,92$ & $-328,35$ & $-45,83$ & $-65,65$ & $-85,54$ & $-209,61$ & $-497,94$ & $-22,24$ & $-21,03$ \\
\hline DP & $\pm 93,22$ & $\pm 250,45$ & $\pm 799,01$ & $\pm 108,3$ & $\pm 80,89$ & $\pm 182,99$ & $\pm 250,72$ & $\pm 851,98$ & $\pm 73,56$ & $\pm 51,6$ \\
\hline
\end{tabular}

Continua... 
Rodrigues ACT, Faganello-Navega FR. Avaliação eletromiográfica dos músculos. Rev Ter Ocup Univ São Paulo. 2016 set./dez.;27(3):278-88.

Tabela 2 - Valores de média e desvio-padrão da porcentagem de ativação eletromiográfica durante o exercício em relação ao repouso de todos os músculos analisados, em todas as situações. Os valores que apresentaram diferença significativa $(p<0,05)$ estão em negrito na tabela. Abd_pol: músculo abdutor curto do polegar; fl_radial: músculo flexor radial do carpo; ext_ulnar: músculo extensor ulnar do carpo

\begin{tabular}{|c|c|c|c|c|c|c|c|c|c|c|}
\hline & \multicolumn{5}{|c|}{ DUAS MÃOS - SEM ESPELHO } & \multicolumn{5}{|c|}{ DUAS MÃOS - COM ESPELHO } \\
\hline & abd_pol & fl_radial & ext_ulnar & bíceps & tríceps & abd_pol & fl_radial & ext_ulnar & bíceps & tríceps \\
\hline MÉDIA & 1,04 & 1,8 & 20,96 & 31,79 & $-38,27$ & $-85,58$ & $-38,35$ & $-12,71$ & 54,9 & $-31,39$ \\
\hline \multirow[t]{4}{*}{ DP } & $\pm 96,81$ & $\pm 99,15$ & $\pm 68,8$ & $\pm 51,43$ & $\pm 80,42$ & $\pm 246,19$ & $\pm 189,5$ & $\pm 125,82$ & $\pm 41,52$ & $\pm 67,93$ \\
\hline & \multicolumn{10}{|c|}{ EXERCÍCIO DE FLEXÃO/EXTENSÃO DE COTOVELO } \\
\hline & \multicolumn{5}{|c|}{ MEMBRO NÃO AFETADO - SEM ESPELHO } & \multicolumn{5}{|c|}{ MEMBRO NÃO AFETADO - COM ESPELHO } \\
\hline & abd_pol & fl_radial & ext_ulnar & bíceps & tríceps & abd_pol & fl_radial & ext_ulnar & bíceps & tríceps \\
\hline MÉDIA & $-89,81$ & $-133,71$ & $-226,16$ & $-20,83$ & $-35,58$ & $-13,69$ & $-167,05$ & $-183,89$ & $-23,09$ & $-22,89$ \\
\hline \multirow[t]{3}{*}{ DP } & $\pm 118,56$ & $\pm 281,57$ & $\pm 551,89$ & $\pm 96,05$ & $\pm 86,16$ & $\pm 48,99$ & $\pm 218,89$ & $\pm 309,22$ & $\pm 75,66$ & $\pm 68,93$ \\
\hline & \multicolumn{5}{|c|}{ DUAS MÃOS - SEM ESPELHO } & \multicolumn{5}{|c|}{ DUAS MÃOS - COM ESPELHO } \\
\hline & abd_pol & fl_radial & ext_ulnar & bíceps & tríceps & abd_pol & fl_radial & ext_ulnar & bíceps & tríceps \\
\hline MÉDIA & $-62,99$ & $-68,2$ & $-77,74$ & 75,8 & 8,27 & $-20,26$ & $-61,2$ & $-373,87$ & 63,89 & 13,68 \\
\hline \multirow[t]{4}{*}{ DP } & $\pm 160,01$ & $\pm 192,69$ & $\pm 292,66$ & $\pm 28,41$ & $\pm 78,5$ & $\pm 97,58$ & $\pm 192,58$ & $\pm 1000,4$ & $\pm 49,89$ & $\pm 52,52$ \\
\hline & \multicolumn{10}{|c|}{ EXERCÍCIO DE PRONAÇÃO/SUPINAÇÃO DE ANTEBRAÇO } \\
\hline & \multicolumn{5}{|c|}{ MEMBRO NÃO AFETADO - SEM ESPELHO } & \multicolumn{5}{|c|}{ MEMBRO NÃO AFETADO - COM ESPELHO } \\
\hline & abd_pol & fl_radial & ext_ulnar & bíceps & tríceps & abd_pol & fl_radial & ext_ulnar & bíceps & tríceps \\
\hline MÉDIA & $-207,37$ & $-87,56$ & $-299,81$ & $-42,84$ & $-27,53$ & $-108,3$ & $-227,43$ & $-455,29$ & $-46,15$ & $-45,77$ \\
\hline \multirow[t]{3}{*}{ DP } & $\pm 276,87$ & $\pm 208,71$ & $\pm 774,69$ & $\pm 118,48$ & $\pm 80,22$ & $\pm 191,51$ & $\pm 263,36$ & $\pm 916,95$ & $\pm 103,31$ & $\pm 46,69$ \\
\hline & \multicolumn{5}{|c|}{ DUAS MÃOS - SEM ESPELHO } & \multicolumn{5}{|c|}{ DUAS MÃOS - COM ESPELHO } \\
\hline & abd_pol & fl_radial & ext_ulnar & bíceps & tríceps & abd_pol & fl_radial & ext_ulnar & bíceps & tríceps \\
\hline MÉDIA & 7,41 & $-77,79$ & 29,2 & 43,82 & $-1,35$ & 40,41 & $-14,62$ & 14,03 & 13,86 & $-11,51$ \\
\hline DP & $\pm 63,53$ & $\pm 244,55$ & $\pm 72,7$ & $\pm 48,3$ & $\pm 74,5$ & $\pm 24,69$ & $\pm 131,4$ & $\pm 78,47$ & $\pm 84,9$ & $\pm 76,19$ \\
\hline
\end{tabular}

O teste ANOVA para medidas repetidas apresentou diferença significativa nas seguintes situações: Músculo abdutor do polegar durante exercício de adução/abdução do polegar $(\mathrm{p}=0,005)$; músculo flexor radial do carpo durante flexão/extensão do punho ( $\mathrm{p}=0,032)$; músculo bíceps braquial durante flexão/extensão de cotovelo $(\mathrm{p}=0,002)$ e pronação/supinação de antebraço $(\mathrm{p}=0,025)$ e músculo tríceps braquial na realização de flexão/extensão de cotovelo $(\mathrm{p}=0,024)$; todas com realização dos exercícios apenas com o membro não acometido pela hemiparesia, com e sem o uso do espelho, sendo, esta, a porcentagem de ativação elétrica durante o exercício em relação ao repouso. Não foi encontrada diferença significativa relacionada ao músculo extensor ulnar do carpo. A Figura 2 ilustra esses dados.

Por fim, na relação agonista/antagonista, foi encontrada diferença estatisticamente significativa entre os músculos bíceps e tríceps braquial, durante a flexão e extensão de cotovelo, estando os dados ilustrados na Figura 3. Não foram encontradas diferenças significativas entre os músculos flexor e extensor do punho avaliados. 


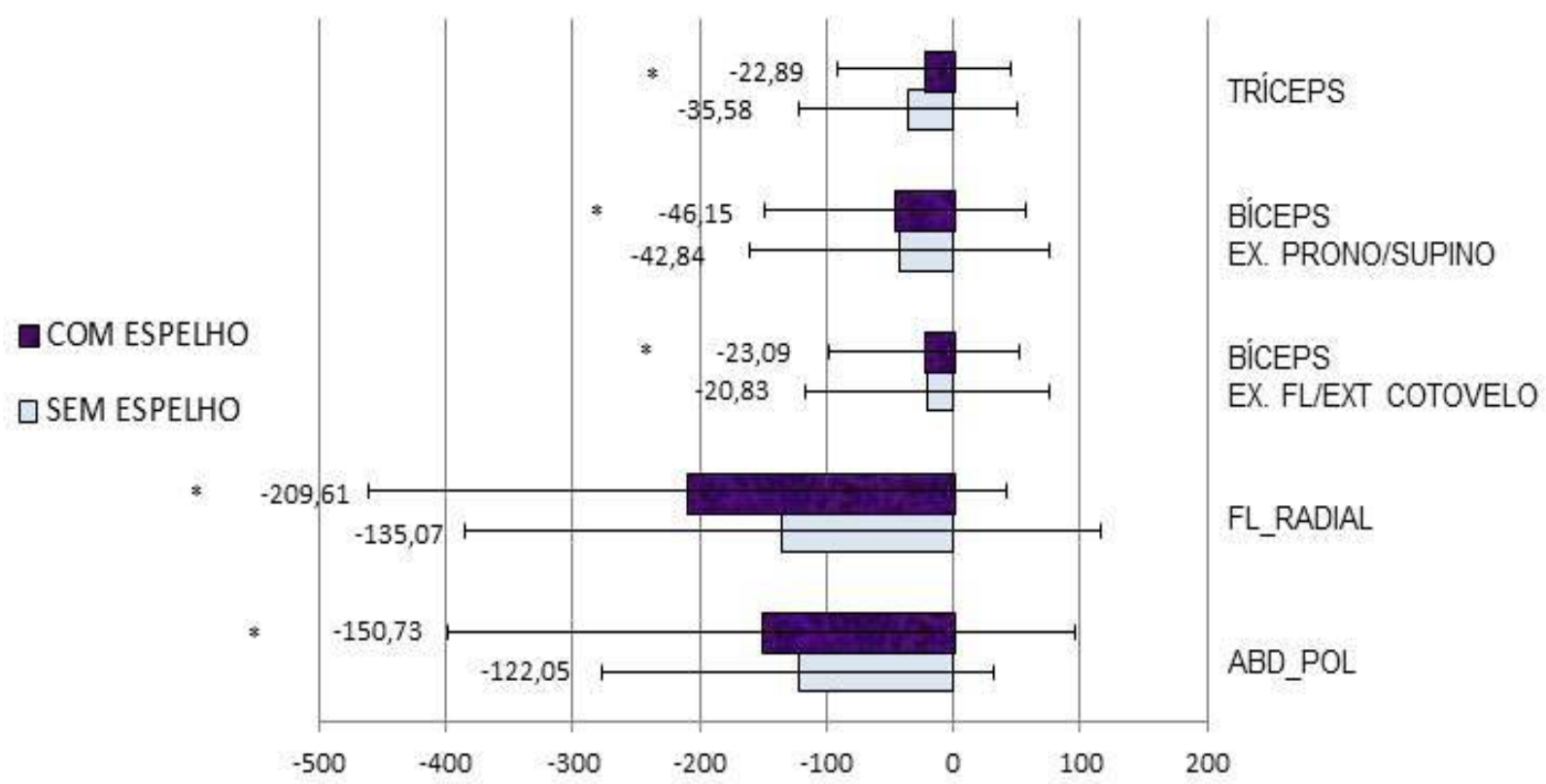

Figura 2 - Dados eletromiográficos que apresentaram diferença estatisticamente significativa, comparando a ativação elétrica com e sem o uso do espelho, realizando os exercícios somente com o membro não comprometido. Porcentagem de ativação durante o exercício em relação ao repouso. Abd_pol: músculo abdutor do polegar; Fl_radial: músculo flexor radial do carpo; Ex. fl/ext cotovelo: exercício de flexão/extensão de cotovelo; Ex. prono/supino: exercício de pronação/supinação de antebraço. *: p<0,05

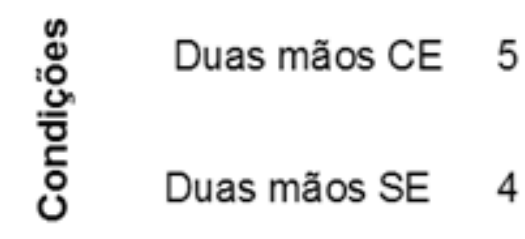

Membro não afetado CE 3

Membro não afetado SE 2

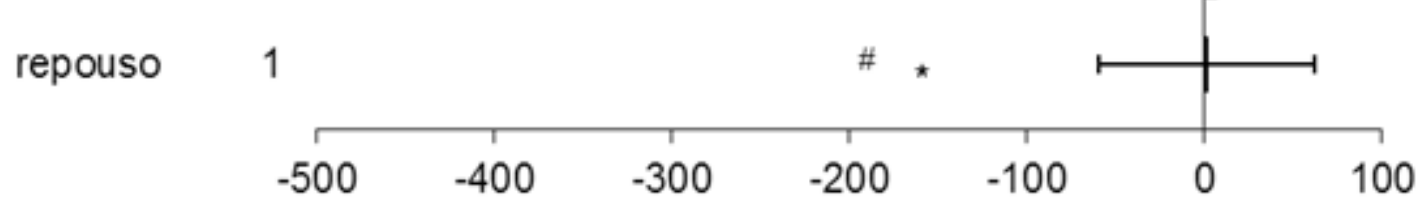

Figura 3 - Relação agonista/antagonista entre os músculos bíceps e tríceps durante todas as condições analisadas. Quando maior que zero, maior ativação do bíceps em relação ao tríceps. Quando menor que zero, maior ativação do tríceps em relação ao bíceps. *: Condições 1 , 2 e 3 diferentes da condição 4; \#: condições 1 e 3 diferentes da condição 5. p<0,05. CE: com espelho; SE: sem espelho 


\section{DISCUSSÃO}

O estudo teve como objetivo analisar a atividade elétrica muscular durante a realização da Terapia do Espelho no membro superior parético de indivíduos com sequela de AVE.

De acordo com os resultados encontrados durante o repouso, foi possível observar maior acometimento da musculatura flexora distal em relação à musculatura flexora proximal, uma vez que a atividade distal foi maior que a proximal, corroborando os achados de Campos et $\mathrm{al.}^{7}$, indicando a predominância da espasticidade na articulação distal do membro superior acometido. Esse resultado justifica o fato de o maior desafio na reabilitação dos pacientes pós-AVE consistir na melhora da mobilidade distal do membro superior, particularmente, na melhora da extensão do punho. Sendo assim, os dados apresentados permitem relacionar a hiperatividade muscular distal do membro superior observada durante o repouso e o maior comprometimento desses músculos na hemiparesia ${ }^{7}$, tendo uma porcentagem menor de ativação em relação à musculatura proximal durante a realização de exercícios ativos.

Não foram encontrados resultados que mostrassem maior aumento do tônus da musculatura flexora distal em relação à musculatura extensora distal durante o repouso, ou seja, maior ativação eletromiográfica do músculo flexor radial em relação ao extensor ulnar do carpo. Assim como o estudo de Campos et al. ${ }^{7}$, era esperado que, pela presença de atividade da musculatura flexora devido à espasticidade de alguns voluntários, o seu antagonista fosse inibido reflexamente, estando, portanto, relaxado; entretanto, este fato não foi observado nos resultados.

Musampa et al. ${ }^{18}$ demonstraram, em um estudo com 10 indivíduos saudáveis e 11 indivíduos pós-AVE, através da EMG de superfície, alteração na regulação do reflexo de estiramento entre agonistas/antagonistas dos grupos musculares flexores e extensores do cotovelo, devido à hipertonia dos flexores dos pacientes com espasticidade e, visto que a regulação do reflexo de estiramento tem sido descrita como mecanismo principal de controle da postura e do movimento em indivíduos saudáveis, sugeriram a avaliação de ambos os membros superiores de indivíduos pós-AVE, e não somente no hemicorpo acometido pela hemiparesia. Os resultados encontrados em nosso estudo corroboram os achados de Musampa et al. ${ }^{18} \mathrm{e}$ de Gerachshenko et al. ${ }^{19}$, nos quais constatou-se atividade muscular antagonista (extensora) durante o repouso no membro superior de indivíduos hemiparéticos.

É importante ressaltar que, diferentemente da pesquisa de Pereira e Araújo ${ }^{6}$ que mostraram, num estudo com 25 indivíduos saudáveis e 25 indivíduos pós-AVE, na condição de repouso, quanto mais severa a espasticidade, maior o sinal EMG do músculo bíceps braquial, outra hipótese para não ter ocorrido maior presença de atividade da musculatura flexora em relação à extensora proximal durante o repouso dos voluntários é devido à pequena pontuação na Escala Modificada de Ashworth dos pacientes, pois, caso fossem selecionados para o estudo voluntários com alto índice de espasticidade, os mesmos não conseguiriam realizar os exercícios propostos, assim como mostram outros autores, que verificaram que a espasticidade influencia para um mau desempenho em testes de destreza manual do membro superior, além de ter relação com a fraqueza muscular, sendo que pacientes com mais espasticidade apresentam maior déficit de força e destreza ${ }^{20,2}$.

Os músculos abdutor curto do polegar, flexor radial do carpo e bíceps braquial apresentaram diminuição da atividade elétrica durante a realização dos exercícios cujos músculos são responsáveis, sendo eles, respectivamente, abdução/ adução do polegar, flexão/extensão de punho e flexão/ extensão de cotovelo quando utilizada a técnica do espelho em comparação ao não uso do espelho, provavelmente, devido à uma maior adequação do tônus muscular, concordando com a hipótese do estudo de que ocorreria alteração na atividade elétrica muscular com a Terapia do Espelho. Acreditamos que essa diminuição da atividade elétrica diz respeito à inibição inter-hemisférica, estudada por Ward e Cohen ${ }^{21}$ e Guimarães et al. ${ }^{22}$, conceito que mostra que, ao realizar movimentação com o membro superior não acometido, reduz-se a atividade motora do lado hemiparético. No caso de nossa pesquisa, a atividade elétrica foi reduzida, pois os pacientes apresentavam certa ativação durante o repouso devido à espasticidade apresentada, embora em níveis mais baixos. Provavelmente, com a intervenção prolongada, seria possível verificar a recuperação motora nesses pacientes, visto que, no nosso estudo, já foi possível observar, através de avaliação e familiarização, uma alteração no padrão eletromiográfico nos músculos estudados, resultado da provável adequação do tônus, requisito necessário para a recuperação motora propriamente dita, assim como citado por Luvizutto e Gameiro ${ }^{23}$.

Essa alteração na atividade elétrica também foi apresentada no músculo bíceps braquial durante o exercício de pronação/supinação, visto que o bíceps, além de ser responsável pela flexão do cotovelo, também auxilia o músculo supinador no exercício de supinação do antebraço.

Por outro lado, os músculos analisados apresentaram menor porcentagem de ativação durante a 
realização de exercícios com o membro contralateral sem o uso do espelho, comparado ao repouso, possibilitando constatar que ocorreu a adequação do tônus através da inibição inter-hemisférica, porém, este fato também ocorreu durante a realização da Terapia do Espelho, onde somente a avaliação e a familiarização não foram suficientes para que os pacientes realizassem de forma efetiva o movimento com o membro hemiparético. Desta forma, acreditamos ser necessário a intervenção com uso da Terapia do Espelho, assim como realizado por pesquisadores como Altschuler et al. ${ }^{10}$ que realizaram a terapia durante 4 semanas; Stevens e Stoykov ${ }^{11} 3$ semanas e Yavuzer et al. ${ }^{12}$ durante 4 semanas.

Foi possível observar, na relação agonista/ antagonista de bíceps e tríceps braquial que houve uma maior ativação eletromiográfica do bíceps em relação ao tríceps durante o repouso, o que era esperado, devido à presença da espasticidade flexora nos pacientes. Durante a realização de exercícios com o membro contralateral, o tônus do bíceps se adequou, reduzindo a atividade e, aumentando, consequentemente, a atividade do tríceps, seu antagonista. Durante a realização de exercícios com ambos os membros superiores, a ativação do tríceps foi consideravelmente maior em relação à ativação do bíceps no exercício de flexão e extensão de cotovelo, concordando com os pressupostos de que a espasticidade colabora para

\section{REFERÊNCIAS}

1. Ares MJJ. Acidente Vascular Cerebral. In: Teixeira, E.; Sauron, F. N.; Santos, L. S. B.; Oliveira, M.C. Terapia Ocupacional na Reabilitação Física. São Paulo: Roca, 2003, cap.1, p. 3-16.

2. Soares AV, Kerscher C, Uhlig L, Domenech SC, Borges NG. Dinamometria de preensão manual como parâmetro de avaliação funcional do membro superior de pacientes hemiparéticos por acidente vascular cerebral. Fisioter Pesqui. São Paulo, 2011;18(4):359-64. DOI: http://dx.doi. org/10.1590/S1809-29502011000400011

3. Barcala L, Collela F, Araújo MC, Salgado ASI, Oliveira CS. Análise do equilíbrio em pacientes hemiparéticos após o treino com o programa Wii Fit. Rev Fisioter Mov. Curitiba. 2011;24(2):337-43. DOI: 10.1590/S0103-51502011000200015.

4. Barnes MP. Spasticity: a rehabilitation challenge in the elderly. Gerontology. 2001;47(6):295-9. um recrutamento inadequado de unidades motoras e, consequentemente, um mau desempenho motor em geral do membro ${ }^{24,25}$.

Como limitação do estudo podemos citar o fato de não ter sido realizada comparação do membro superior acometido pela hemiparesia com o lado contralateral dos voluntários, não ter sido verificada a dominância e não ter sido realizada avaliação da sensibilidade dos voluntários.

Faz-se necessária a realização de futuros estudos que comparem a atividade elétrica muscular do membro superior de indivíduos pós-AVE antes e após intervenção com uso da Terapia do Espelho, além da utilização de outro instrumento de avaliação funcional aliado à eletromiografia de superfície.

\section{CONCLUSÃO}

Diante dos resultados apresentados, foi possível observar alteração na atividade eletromiográfica dos músculos do membro superior hemiparético de indivíduos pós-AVE com o uso da Terapia do Espelho, porém, ainda é necessária a realização de estudos que comparem estes dados pré e pós-intervenção com o uso da técnica, em busca de uma recuperação motora mais efetiva do membro acometido.

5. Alves RS. Terapia espelho: atividade elétrica e força muscular após aplicação de um protocolo de tarefas motoras. São José dos Campos: Universidade do Vale do Paraíba, 2012.

6. Pereira AC, Araújo RC. Estudo sobre a eletromiografia de superfície em pacientes portadores de espasticidade. Rev Bras Fisioter. 2002;6(3):127-34. Disponível em: http:// www.rbf-bjpt.org.br/files/v6n3/v6n3a04.pdf.

7. Campos TF, Ribeiro TS, Melo LP, Farias I. MA, Macedo LRD, Dantas LTAB, Oliveira DC, Brasileiro JS. Análise eletromiográfica do músculo espástico de pacientes hemiparéticos pré e pós-intervenção fisioterapêutica. Ter Man. 2012;10(48):148-53. DOI: 10.9736/TerMan.v10.n49.93.

8. Song R, Tong KY. EMG and kinematic analysis of sensorimotor control for patients after stroke using cyclic voluntary movement with visual feedback. J Neuro Eng Rehab. 2013;10(18):1-9. DOI:10.1186/1743-0003-10-18. 
9. Machado S, Velasques B, Paes F, Cunha M, Basile LF, Budde H, Cagy M, Piedade R, Ribeiro P. Terapia-espelho aplicada à recuperação funcional de pacientes pós-acidente vascular cerebral. Rev Neurocienc. 2011;19(1):171-5. Disponível em: http://www.revistaneurociencias.com.br/edicoes/2011/ RN1901/opiniao\%20e\%20rev\%20aberta/586\%20opiniao. pdf.

10. Altschuler EL, Wisdom SB, Stone L, Foster C, Galasko D, Llewellen DME, Ramachandran VS. Rehabilitation of hemiparesis after stroke with a mirror. Lancet. 1999;353:2035-6. DOI: 10.1016/S0140-6736(99)00920-4.

11. Stevens JA, Stoykov MEP. Simulation of bilateral movement training through mirror reflection: a case report demonstrating an Occupational Therapy technique for hemiparesis. Topics Stroke Rehabil. 2004;11(1):59-66. DOI: 10.1310/GCFE-QA7A-2D24-KHRU.

12. Yavuzer G, Selles R, Sezer N, Sutbeyaz S, Bussman JB, Koseoglu F, Atay MB, Stam HJ. Mirror therapy improves hand function in subacute stroke: a randomized controlled trial. Arch Phys Med Rehabil. Chicago. 2008;89:393-8. DOI: 10.1016/j.apmr.2007.08.162.

13. Winstein CJ, Miller JP, Blanton S, Taub E, Uswatte G, Morris D, Nichols D, Wolf S. Methods for a multisite randomized trial to investigate the effect of constraintinduced movement therapy in improving upper extremity function among adults recovering from a cerebrovascular stroke. Neurorehabil Neural Repair. 2003;17(3):137-52. DOI: doi: $10.1177 / 0888439003255511$

14. Palavro BEM, Schuster RC. Efeitos da terapia de contensão induzida adaptada na funcionalidade e qualidade de vida de pacientes hemiparéticos. Rev Fisioter S Func. Fortaleza, 2013;2(2):51-60.

15. Bohannon RW, Smith MB. Interrater reliability of a modified ashworth scale of muscle spasticity. Phys Ther. 1987;67(2):206-7.

16. Minutoli VP, Delfino M, Freitas STT, Lima MO, Tortoza C, Santos CA. Efeito do movimento passivo contínuo isocinético na hemiplegia espástica. Acta Fisiátr. 2007;14(3):142-8.

Recebido em: 24.04.16

Aceito em: 12.09.16
17. Mogk JPM, Keir PJ. Crosstalk in surface electromyography of the proximal forearm during gripping tasks. J Electromyogr Kinesiol. 2003;13(1):63-71. DOI: http:// dx.doi.org/10.1016/S1050-6411(02)00071-8

18. Musampa NK, Mathieu PA, Levin MF. Relationship between stretch reflex thresholds and voluntary arm muscle activation in patients with spasticity. Exp Brain Res. 2007;181(4):579-93. DOI: 10.1007/s00221-007-0956-6

19. Gerachshenko T, Rymer WZ, Stinear JW. Abnormal corticomotor excitability assessed in biceps brachii preceding pronator contraction post-stroke. Clin Neurophysiol. 2008;119(3):683-92. DOI: 10.1016/j.clinph.2007.11.004

20. Smania N, Paoluzzi S, Tinazzi M, Manganotti P, Fiaschi A. Active finger extension: a simple movement predicting recovery of arm function in patients with acute stroke. Stroke. 2007;38(3):1088-90. DOI: 10.1161/01. STR.0000258077.88064.a3

21. Ward NS, Cohen LG. Mechanisms underlying recovery of motor function after stroke. Arch Neurol. 2004;61(12):18448. DOI: 10.1001/archneur.61.12.1844

22. Guimarães CM, Brasil-Neto JP, Oliveira L, Valencia CEU. Desempenho motor em hemiparéticos após treino de relaxamento do membro superior não afetado. Rev Neurocienc. 2011;19(3):496-503.

23. Luvizutto GJ, Gameiro MO. Efeito da espasticidade sobre os padrões lineares da marcha em hemiparéticos. Fisioter Mov. 2009;24(4):705-12. http://dx.doi.org/10.1590/S010351502011000400015

24. Barker RN, Brauer S, Carson R. Training-induced changes in the pattern of triceps to biceps activation during reaching tasks after chronic and severe stroke. Exp Brain Res. 2009;196(4):483-96. doi: 10.1007/ s00221-009-1872-8

25. Hu XL, Tong KY, Wei XJ, Rong W, Susanto EA, Ho SK. The effects of post-stroke upper-limb training with an electromyography (EMG) -driven hand robot. J Electromyogr Kinesiol. 2013 Oct;23(5):1065-74. doi: 10.1016/j.jelekin.2013.07.007. Epub 2013 Aug 7. 